\title{
Predicción de la composición química de harina de sangre bovina mediante Espectroscopia de Reflectancia en el Infrarrojo Cercano (NIRS)
}

\section{Prediction of the chemical composition of bovine blood flour through Near Infrared Spectroscopy (NIRS)}

Flor Mejía ${ }^{1, a, *}$, Wilmer Bernal ${ }^{1, b}$, José Zamora ${ }^{1, c}$, Ives Yoplac ${ }^{1, d}$

${ }^{1}$ Facultad de Ingeniería Zootecnista, Agronegocios y Biotecnología, Universidad Nacional Toribio Rodríguez de Mendoza de Amazonas, Chachapoyas, Perú.

a M.Sc., $\bigotimes_{\text {flor.mejia@untrm.edu.pe, (1D https://orcid.org/0000-0002-1851-1285 }}$

b M.Sc., $\mathbb{}_{\text {wilmer.bernal@untrm.edu.pe, (D) https://orcid.org/0000-0003-3482-8466 }}$

c M.Sc., $\bigotimes$ jose.zamora@untrm.edu.pe, (DD https://orcid.org/0000-0003-3362-9609

cPh.D., 囚 $\underline{\text { ives.yoplac@untrm.edu.pe, (D) https://orcid.org/0000-0001-9524-1584 }}$

* Autor de Correspondencia: Tel. +51 935990754

http://dx.doi.org/10.25127/riagrop.20212.675

http://revistas.untrm.edu.pe/index.php/RIAGROP revista.riagrop@untrm.edu.pe

Recepción: 22 de enero 2021

Aprobación: 23 de marzo 2021

Este trabajo tiene licencia de Creative Commons. Attribution-NonCommercial-ShareAlike $\quad 4.0$ International Public License - CC-BY-NC-SA 4.0

\section{Resumen}

El objetivo de la investigación fue predecir la composición química de harina de sangre bovina (HSB), mediante espectroscopia de reflectancia en el infrarrojo cercano (NIRS), de las fracciones: Humedad $(\mathrm{H})$, Proteína Bruta (PB), Extracto Etéreo (EE) y Ceniza (C). Se recolectó sangre de ganado vacuno de 1.5 a 8 años de edad, del centro de beneficio municipal de Chachapoyas. Se prepararon 30 muestras de HSB previa cocción a fuego lento por una hora y secado en una estufa a $60{ }^{\circ} \mathrm{C}$. Se tomaron espectros en NIRS 2500XL (Unity Scientific, USA) y de las muestras analizadas en laboratorio por el método proximal, se obtuvo los químicos de referencia para generar el modelo de predicción con el software UCAL Model, por la primera derivada y los métodos de regresión RLM/ACP, RLM y RCP y se obtuvo el coeficiente de determinación R2 de (0.93, 0.83 y 0.82); $(0.74,0.78,0.19)$; $(0.67,0.79,0.39)$ y $(0.69,0.64,0.19)$ y el error estándar de calibración EEC de $(0.38,0.38,0.38)$; $(1.39,1.27,1.39)$; 
$(0.04,0.05,0.04)$ y $(0.36,0.35,0.65)$; para $\mathrm{H}, \mathrm{PB}, \mathrm{EE}$ y C respectivamente. Por tanto, el modelo RLM tiene potencial en la calibración de la predicción de la de la harina de sangre bovina.

Palabras Clave: Calibración, espectro, NIRS, predicción, validación del modelo.

\begin{abstract}
The purpose of the research was to predict the chemical composition of bovine blood flour (BBM) by means of the reflectance of near infrared spectroscopy (NIRS), of the fractions: Humidity (H), Crude Protein (CP), Ethereal Extract (EE) and Ash (A). Bovine blood was collected from 1.5 to 8 years of age, of the Chachapoyas municipal benefit center. 30 samples HSB were prepared from previous simmering for one hour and drying in an oven at $60^{\circ} \mathrm{C}$, spectra were taken in NIRS 2500XL (Unity Scientific, USA), and the samples analyzed in the laboratory by the proximal method obtaining the chemicals of Reference to generate the model of prediction with the software UCAL Model, by the first derivative and the regression methods MLR/PCA, MLR and PCR, obtaining the coefficient of determination R2 of $(0.93,0.83$ and 0.82$)$; $(0.74,0.78,0.19)$; $(0.67$, $0.79,0.39)$ and $(0.69,0.64,0.19)$ and the standard EEC calibration error of $(0.38,0.38,0.38) ;(1.39,1.27,1.39)$; $(0.04,0.05,0.04)$ and $(0.36,0.35,0.65)$; For H, PB, EE and C respectively. Therefore, the MLR model has potential in the calibration of the prediction of bovine blood flour.
\end{abstract}

Keywords: Calibration, spectrum, NIRS, prediction, validation model.

\section{INTRODUCCIÓN}

Los equipos tradicionalmente usados para determinar la composición química de insumos, o productos agroalimenticios, presentan inconvenientes como el tiempo prolongado, costos elevados y la utilización de químicos peligrosos que genera efluentes como desechos, ácidos, detergentes y gases irritantes, peligroso para los operarios y son fuentes de contaminación ambiental (Cozzolino et al. 2005).

NIRS (Near Infrared Spectroscopy) es una técnica de análisis rápido, no destructiva, multiproducto y, por su fiabilidad, es cada vez más utilizada en el control de calidad de insumos y productos. Además, requiere de muestras pequeñas, tiempo corto de análisis, sin uso de reactivos y no destruye la muestra. Esto se refleja en los costos más bajos (80\% de los equipos tradicionales) (Brown y Moore,
1987; Zhang y Su, 2014 y Asekova et al. 2016). Sandoval et al. (2008) mencionan que NIRS permite determinar la composición química precisa a bajo coste y en corto tiempo, pero es necesario generar o ajustar modelos matemáticos de predicción, a partir del análisis químico de laboratorio realizados por métodos tradicionales (Mejía et al., 2016 y Mejía et al, 2019). NIRS basa su predicción en absorción que son expresadas como sobretonos o picos, mediante grupos funcionales como: $\mathrm{C}-\mathrm{H}, \mathrm{O}-\mathrm{H}$, $\mathrm{N}-\mathrm{H}$ y también $\mathrm{S}-\mathrm{H}$ y $\mathrm{C}-\mathrm{O}$, los más comunes constituyen agua, proteínas lípidos y carbohidratos, que responden a la radiación en el infrarrojo cercano (Jiménez, 2007 y Sandoval et al., 2008).

Actualmente, los centros de beneficio generan desechos como: sangre, vísceras, contenido ruminal y demás efluentes a partir del beneficio de animales que, generalmente, son enviados a la red de alcantarillado, sin ningún tratamiento 
previo y generan problemas de contaminación ambiental (Terevinto y Chiesa, 2008 y Moreta, 2012). La sangre representa del 7 a $9 \%$ del peso vivo del animal (Madrid, 1999) y es un potencial insumo de alta calidad para alimentación de especies domésticas. La HSB contiene alta concentración de proteína (FEDNA, 2012 y Ricci, 2012). Por esta razón, se hace necesario predecir la composición química de harina de sangre bovina mediante la tecnología NIRS y generar un valor agregado a la sangre de los centros de beneficio.

\section{MATERIALES Y MÉTODOS}

Se utilizaron muestras de sangre procedente de 20 vacunos de 1.5 a 8 años de edad. Los análisis químicos y de predicción se realizaron en la Universidad Nacional Toribio Rodríguez de Mendoza - UNTRM y en Laboratorio de Nutrición Animal y Bromatología de Alimentos de La UNTRM.

\subsection{Método experimental para la obtención de harina de sangre}

\subsubsection{Recolección de la sangre}

La sangre fue obtenida directamente de la vena yugular, inmediatamente después del degüello. Posteriormente se colocó en un balde de 5 litros con $15 \mathrm{~g}$ de citrato de sodio como anticoagulante. Luego, se llevó al campus universitario para realizar proceso de deshidratación.

\subsubsection{Deshidratación de la sangre}

Este proceso se realizó en ollas de $15 \mathrm{~L}$ de capacidad. La sangre fue concentrada $y$ deshidratada, con el uso de una cocina a gas, a una temperatura $80^{\circ} \mathrm{C}$ por una hora.

\subsubsection{Enfriamiento y secado}

El enfriamiento se realizó en bandejas de metal, y el secado, en una estufa a $60^{\circ} \mathrm{C}$, por un tiempo aproximado de dos días.

\subsubsection{Molido}

Las muestras secas se trituraron en un molino de laboratorio. Luego se envasó $200 \mathrm{~g}$ en bolsas de polietileno que fueron selladas con una selladora manual hasta completar un total de 30 muestras necesarias para la calibración.

\subsubsection{Almacenamiento}

Las muestras se almacenaron a temperatura ambiente hasta realizar su análisis químico respectivo, previo a la obtención de sus espectros.

\subsubsection{Análisis químico de referencia de la harina de sangre}

El análisis químico de referencia se realizó de manera empírica, con equipos tradicionales en el laboratorio de Nutrición Animal de La UNALM. Considerándose un análisis proximal constituido por la Humedad $(\mathrm{H})$, Proteína Cruda (PC), Fibra cruda (FC), Extracto Etéreo (EE) y Cenizas (C) mediante el método de la AOAC (2005).

\subsection{Metodología para la predicción de la composición de la harina de sangre}

\subsubsection{Obtención de espectros}

Se utilizó las mismas muestras a las que se le evaluó el análisis químico de referencia, en el equipo NIRS (Unity Scientific, USA, 2500XL). Se colocó $80 \mathrm{~g}$ de muestra en celdas de cuarzo, tapada con una cubierta $4 \mathrm{~cm}$ de diámetro por 1 $\mathrm{cm}$ de altura. Además, se tomó los espectros en el rango de 1100 a $2500 \mathrm{~nm}$ y se guardó cada espectro indicando su respectiva identificación. 
Al terminar el procedimiento se creó una carpeta llamada harina de sangre, posteriormente se copió los espectros del quipo NIRS y se llevó a un computador para realizar la calibración respectiva.

\subsubsection{Calibración}

Se utilizó con el Software UCAL Model, para la cual los resultados del análisis químico de referencia obtenido de cada muestra, se ordenaron en una plantilla de Excel, con su respectiva identificación, y los espectros obtenidos en el NIRS. Luego, fueron llevados al computador que contenía el software UCAL Model donde se procedió a la calibración, utilizando funciones estadísticas para desarrollar los modelos matemáticos.

El proceso de calibración se realizó empleando diferentes modelos de regresión:

- Regresión Lineal Múltiple asociada a un Análisis de Componentes principales (MLR/ACP)

- Regresión Lineal Múltiple (MLR)

- Análisis de regresión por componentes principales (RCP)

\subsection{Estadísticos evaluados para determinar el mejor modelo de predicción.}

Desde el punto de vista estadístico, que se utiliza para evaluar ecuaciones NIRS no es totalmente estandarizado por NIRS, fueron más frecuente en trabajos publicados y software que difieran a veces los estadísticos (Wells, 2006) entre los utilizados son: el coeficiente de determinación $\left(\mathrm{R}^{2}\right)$ (Valenciaga, 2006). Walpole et al. (1999) definen que el $\mathrm{R}^{2}$ indica cual es la proporción de la variación total en la respuesta del valor predicho. Asimismo, Westerhaus (1989) explica que un $\mathrm{R}^{2}$ de 0 indica que no hay relación entre ambos métodos. Asimismo, un $\mathrm{R}^{2}$ igual a 1 indica un ajuste perfecto con un error estándar de calibración de 0 . Además, un valor bajo de $\mathrm{R}^{2}$ indica que los valores de laboratorio son imprecisos.

Otro estadístico evaluado fue el error estándar de calibración (EEC). Sobre esto, Davies y Grant (1987) mencionan que permite comparar calibraciones generadas a partir de un mismo grupo de datos e indican la incertidumbre de la calibración. Además, Westerhaus (1989) indica que para evaluarlo se realiza una comparación con el error estándar del método de referencia. Se aceptó hasta el doble del error estándar del método de referencia.

\section{RESULTADOS Y DISCUSIÓN}

\subsection{Composición química de las muestras}

Los resultados obtenidos de la composición química de harina de sangre bovina obtenida por los métodos de referencia son presentados en la tabla 1. Las muestras presentan un rango para humedad de 6.82 a $13.83 \%$; proteína, 73.27 a $87.81 \%$; extracto etéreo 0.15 a $0.68 \%$ y cenizas de 2.01 a $4.40 \%$. Se obtuvo un amplio rango de variación en el set de las 30 muestras utilizadas en la calibración. Los valores de la composición nutricional de la harina de sangre fue para humedad $9.20 \%$, proteína $81.71 \%$, grasa $0.30 \%$ y cenizas $3.20 \%$, concuerdan con los resultados de obtenidos por Santiago et al. (2011), descrito en las tablas brasileras para aves y cerdos de composición de alimentos y requerimientos nutricionales siendo de $7.1 \%, 83.5 \%, 0.46 \%$ y $3.4 \%$ respectivamente. Los rangos del porcentaje humedad son superiores a las obtenidos por Ockerman (2000). También, la Fundación Española para el Desarrollo de la Nutrición Animal [FEDNA] (2012) muestra resultados similares de $8 \%, 87 \%, 0.8 \%$ y $3.5 \%$. 
Estas diferencias se deben al diferente procedimiento realizado para la obtención de dicho producto. Sin embargo, Beltrán y Perdomo (2007), Cifuentes (2007) y Zamora (2016) muestran resultados inferiores en proteína, pero superiores es extracto etéreo y cenizas. Dichos resultados podrían deberse a los diferentes tipos de proceso de obtención de harina de sangre y uso de diferente tipo de anticoagulante.

Tabla 1. Composición química de harina de sangre obtenida por los métodos de referencia

\begin{tabular}{lcccc}
\hline Nutriente & Prom. & Mín. & Máx. & DS $^{1}$ \\
\hline Humedad & 9.20 & 6.82 & 13.83 & 1.46 \\
Proteína & 81.71 & 73.27 & 87.81 & 2.66 \\
Extracto E & 0.30 & 0.15 & 0.68 & 0.10 \\
Cenizas & 3.20 & 2.01 & 4.40 & 0.63 \\
\hline
\end{tabular}

1Desviación Estándar

\subsection{Perfiles espectrales de las muestras de harina de sangre bovina}

Los perfiles se colectaron en el equipo NIRS, con un rango de longitud de onda 1100 a $2500 \mathrm{~nm}$ y se obtuvieron en las absorbancias de cada una de las muestras donde existe variabilidad. Todo esto fue favorable para el desarrollo de un modelo, pero, esto no es el caso para este modelo debido a la baja cantidad de muestras, como se observa en la figura 1.

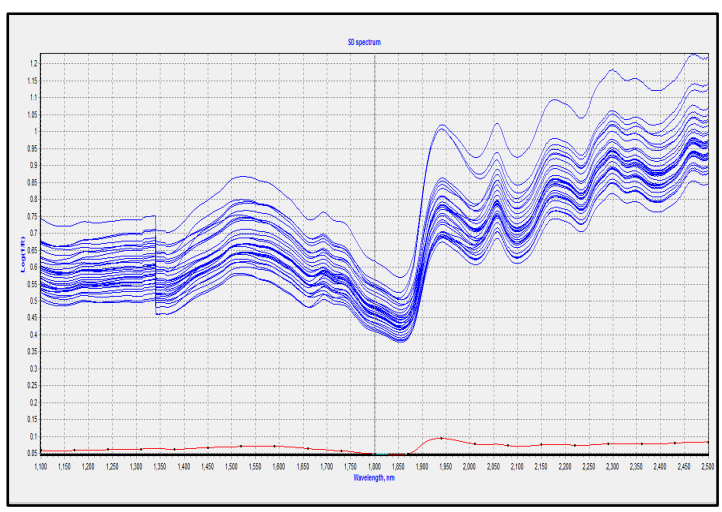

Figura 1. Perfiles espectrales de harina de sangre, obtenido en el equipo NIRS.

\subsection{Creación del modelo predictivo}

Las ecuaciones de calibración se muestran en la tabla 2. En el parámetro de humedad se obtuvo una mejora calibración con el modelo RLM/ACP con un $\mathrm{R}^{2}$ de 0.93 . En proteína $\mathrm{y}$ extracto etéreo el mejor modelo de calibración fue RLM con un $\mathrm{R}^{2}$ de 0.78 y 0.79 respectivamente. En el parámetro de ceniza no se obtuvo calibraciones aceptables en ninguno de los modelos.

Tabla 2. Regresiones utilizadas para la composición química de harina de sangre de 30 muestras

\begin{tabular}{llcccc}
\hline $\begin{array}{c}\text { Método de } \\
\text { regresión }\end{array}$ & & H & PB & EE & C \\
\hline \multirow{2}{*}{ RLM/ACP } & $\mathrm{N}^{2}$ & 29 & 29 & 29 & 29 \\
& $\mathrm{SD}^{3}$ & 1.47 & 2.75 & 0.08 & 0.65 \\
& $\left(\mathrm{R}^{2}\right)^{4}$ & 0.93 & 0.74 & 0.67 & 0.69 \\
& $\mathrm{EEC}^{5}$ & 0.38 & 1.39 & 0.04 & 0.36 \\
$\mathrm{RLM}$ & $\mathrm{N}^{2}$ & 28 & 27 & 28 & 24 \\
& $\mathrm{SD}^{3}$ & 1.04 & 2.7 & 0.11 & 0.57 \\
& $\left.\mathrm{(R}^{2}\right)^{4}$ & $\mathbf{0 . 8 3}$ & $\mathbf{0 . 7 8}$ & $\mathbf{0 . 7 9}$ & $\mathbf{0 . 6 4}$ \\
& $\mathrm{EEC}^{5}$ & 0.38 & 1.27 & 0.05 & 0.35 \\
$\mathrm{RCP}$ & $\mathrm{N}^{2}$ & 29 & 27 & 27 & 29 \\
& $\mathrm{SD}^{3}$ & 1.47 & 1.86 & 0.06 & 0.65 \\
& $\left(\mathrm{R}^{2}\right)^{4}$ & 0.82 & 0.19 & 0.39 & 0.19 \\
& $\mathrm{EEC}^{5}$ & 0.38 & 1.39 & 0.04 & 0.65 \\
\hline
\end{tabular}

${ }^{1}$ RLM/PCA - Regresión lineal múltiple asociado a un análisis de componentes Principales, RLM - Regresión lineal múltiple y RCP - regresión por componentes principales

${ }^{2}$ Número de muestra de calibración

${ }^{3}$ Desviación estándar

${ }^{4}$ Coeficiente de determinación de la calibración

${ }^{5}$ Error estándar de calibración

La mejor ecuación fue obtenida mediante el modelo de regresión lineal múltiple ya que presenta los mejores estadísticos evaluados para proteina y extracto etereo. En la figura 2 y 3 se muestra la relacion que hay entre el analisis quimico de referencia y la predicción mediante NIRS para proteína y grasa respetivamente.

El R $\mathrm{R}^{2}$ obtenido para $\mathrm{H}, \mathrm{P}, \mathrm{EE}$ y $\mathrm{C}$ fue de 0.83 , $0.78, \quad 0.79$ y 0.64 respectivamente fueron semejantes a los encontrados por García et al. (2013) en la determinación de proteína en trigo obteniendo un $\mathrm{R}^{2}$ de 0.99 , dicho producto fue mayor el $\mathrm{R}^{2}$ por el número de muestras evaluadas y también por el producto. Asimismo, Cozzolino (2002) evaluó alimento 
para animales (400 muestras) con diferentes harinas proteicas tanto animales como vegetales considerando la harina de sangre en una pequeña proporción y obtuvo el $\mathrm{R}^{2} \mathrm{de} 0.98$ para proteína y de 0.92 para cenizas al igual que la investigación anterior los resultados son afines ya que los productos son distintos y el número de muestra es relativamente grande. Asimismo, Vivar (2009) desarrolló predicciones para harina de sangre de ave y reportó resultados superiores a los obtenidos en este estudio, en los parámetros de humedad, proteína bruta, extracto etéreo y cenizas reportaron un $\mathrm{r}^{2}$ superior a 0 . Estas diferencias se deberían al tipo de harina de sangre y al número de muestras evaluadas (180 muestras).

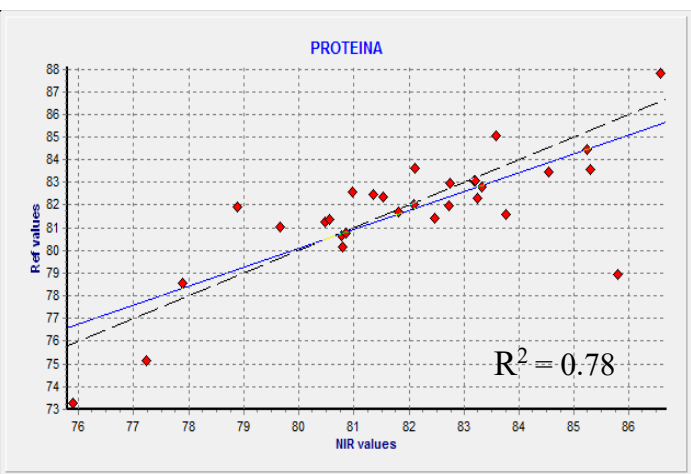

Figura 2. Relación entre el análisis químico de referencia y la predicción mediante NIRS para proteína.

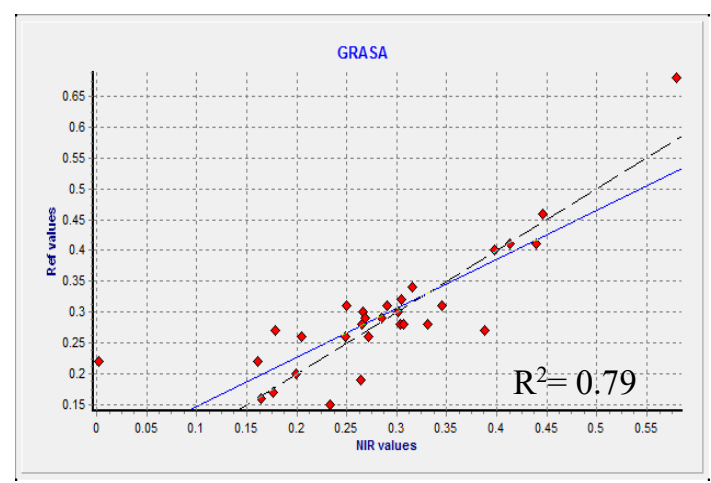

Figura 3. Relación entre el análisis químico de referencia y la predicción mediante NIRS para extracto etéreo.

Los resultados de ceniza no son buenos para realizar la calibración ya que muestra mucha dispersión de los datos como se muestra en la figura 4. Dichos resultados podrían deberse al bajo número de muestras y al tipo de modelo de regresión utilizado para realizar la calibración.

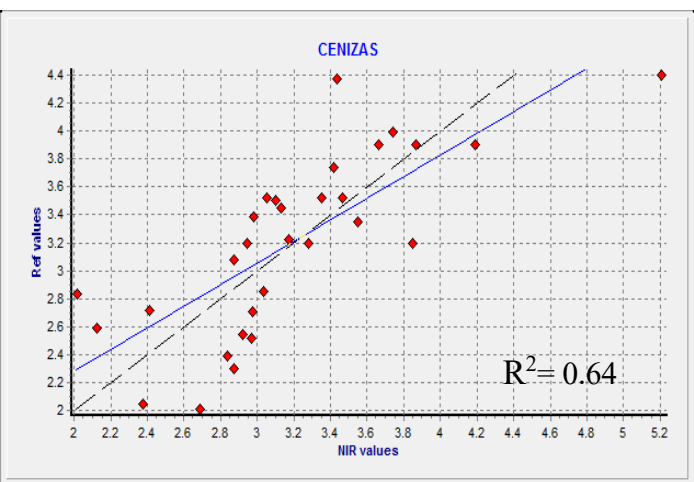

Figura 4. Relación entre el análisis químico de referencia y la predicción mediante NIRS para cenizas (Modelo MLR).

\section{CONCLUSIONES}

Los modelos de regresión RLM/ACP y RLM reportaron para los parámetros de humedad y proteína buenas calibraciones, con un $\mathrm{r}^{2}$ superior a 0.7. El Modelo de regresión PCR solo presento una buena calibración para el parámetro de humedad.

El modelo RLM fue el mejor modelo de calibración presento resultados superiores a 0.7 obtuvo en los parámetros de humedad, proteína cruda y extracto etéreo.

El parámetro de Ceniza no se obtuvo ninguna calibración aceptable en ninguno de los modelos.

\section{Agradecimientos:}

Al Instituto de Investigación en Ganadería y Biotecnología (IGBI), y Laboratorio de Nutrición Animal y Bromatología de los alimentos de la Universidad Nacional Toribio Rodríguez de Mendoza de Amazonas.

\section{Referencias}

[AOAC] Association of Official Analytical Chemists. 2005. Official Methods of Analysis. 18 th ed. Washington, USA. 
Asekova S, Han SI, Choi HJ, ParkSJ, Shin DH, Kwon CH, Lee JD.2016. Determination of forage qualityby nearinfrared reflectance spectros-copy in soybean. Turk J Agric For 40:45-52. doi: 10.3906/tar-1407-33

Beltrán y Perdomo, 2007. Aprovechamiento de la sangre de bovino para la obtencion de harina de sangre y plasma sanguineo en el matadero Santa Cruz de Malambo Atlantico. Universidad de la Salle, Colombia. 193pCifuentes, Oscar I. 2007. Proceso artesanal de producción de harina de sangre de bovino, componentes de la sangre, procesamiento. Universidad de Caldas, Colombia.

Brown, W. F. y Moore, J. E. (1987). Analysis of Forage research Samples utilizing a combination of wet Chemistry and Near Infrared Reflectance Spectroscopy. Journal of Animal Science. doi.org/10.2527/jas1987.641271x

Cozzolino, D. (2002). Uso de la espectroscopia de reflectancia en el infrarrojo cercano (NIRS) en el análisis de alimentos para animales. Agrociencia. Vol. VI. INIA La Estansuela. Colonia - Uruguay.

Cozzolino, D., Fassio, A. \& Fernández, E. 2005. Uso de la espectroscopia de reflectancia en el infrarrojo cercano para el análisis de calidad de ensilaje de maíz. Agric. Téc. 63:387

Davies, A. M. y Grant, A. (1987). Near infrared analysis of food. Journal Food Science.

Fundación Española para el Desarrollo de la Nutrición Animal. 2012. Tablas de composición nutricional de insumos.

García, M., García, J. y Barro, F. 2013. Determinación de parámetros de calidad de variedades de trigo comuy bajo contenido en epítopos de celiaquía mediante NIR. Departamento de Mejora Genética Vegetal. Córdoba.

Givens, D. I., De boever, J. L y Deaville, E. R. (1997). The principles, practices and some future applications of near infrared spectroscopy for predicting the nutritive value of foods for animals and humans. Nutrition Research Rewiews.

Madrid, A. (1999). Aprovechamiento de los subproductos cárnicos.

Mejía, F. Bernal, W. y Yoplac, I. 2016. Predicción de la composición química de harina de sangre bovina mediante espectroscopia de reflectancia en el infrarrojo cercano (NIRS). Tesis para optar el título de Ingeniero Zootecnista - UNTRM.

Mejía, F. Yoplac, I. Bernal, W y Castro, W. 2019. Evaluación de modelos de predicción de composición química y energía bruta de kikuyo (Pennisetum clandestinum) usando espectroscopía en infrarrojo cercano (NIRS).
Rev Inv Vet Perú 30(3): 1068-1076 http://dx.doi.org/10.15381/rivep.v30i3.16598

Moreta, M. (2012). Diseño de un secador tipo túnel para la deshidratación de la harina de sangre en el camal frigorífico de Riobamba.

Ockerman, H. 2000. Industrializacion de subproductos. 2da Ed. Barcelona-España. Edit Satrling. 13-19p.

Reeves, J., Blosser, T., Balde, A., Glenn, B. y Vandersal, J. (1991). Near infrared spectroscopy analysis of forage samples digested in situ (nylon bag). J. Dairy Science

Ricci, O. 2012. Harina de Sangre". (En línea). Consultado, 12 de marzo. 2021. Formato HTML. Disponible en http://www.engormix.com/

Sandoval, L. A., Bueso-Uclés, F. J., Vélez-Nauer, M. (2008). Predicción nutricional para pastos tropicales por espectroscopía de reflectancia en el infrarrojo cercano. Universidad de Costa Rica. Agronomía Mesoamericana.

Santiago, H., Teixeira, L., Lopes, J., Cezar, P., Flávia, R., Lopes, D., Soares, A., Toledo, S. y Euclides, R. 2011. Tablas Brasileras para Aves y Cerdos, Composición de Alimentos y Requerimientos Nutricionales. 3a Universidad Federal de Viçosa. Brasil.

Shenk, J. y Werterhaus, M. (1994). The application of Near Infrared Reflectance Spectroscopy (NIRS) to forage analysis. Ed. In Fahey, G. C.

Terevinto, A. y Chiesa, Carolina, 2008. Subproductos Agroindustriales de Origen Animal. (En Línea). Consultado el 27 de marzo de 2009. Disponible en: http://www.fagro.edu.uy/ nutanimal/Chiesa\%20Ter evinto.SUB\%20ANIMALES.2008

Walpole, R., Myers, R. y Myers, S. (1999). Probabilidad y estadística par ingenieros. Traducido por Cruz, R. 6aa. ed. México.

Wells, G. (2006). Spectroscopía de reflectancia en el infrarrojo cercano (NIRS) en el análisis cuantitativo y cualitativo de carne de cordero. Tesis para obtener el grado de magíster en producción animal Universidad Austral de Chile

Westerhaus, M. (1989). Equation development. Analisys of forage quality. Department of Agriculture- USA.

Zamora, S. J. 2016. Rendimiento y composición corporal de cuyes (Cavia porcellus) suplementados con tres niveles de harina de sangre bovino (Bos taurus) procesada artesanalmente. Universidad Nacional de Trujillo, Perú. 87p.

Zhang C, Su J. 2014. Application ofnear infrared spectroscopy to the analysisand fast quality assessment of traditional Chinese medicinal products. ActaPharmaceutica Sinica 4: 182-192. doi:10.1016/j.apsb.2014.04.001 